\title{
A Step of Mobile Ad-Hoc On-demand Routing Protocols towards 4G Cellular Networks
}

\author{
Jahangir khan, Nazir Shah Khattak, Hamid Jan \\ Department of computer Science \& IT \\ Sarhad University of science and Information Technology Peshawar 25000 Pakistan
}

\begin{abstract}
In the competition of this modern technology the aim of Mobile Ad-Hoc networking is to provide efficient communication in wireless technology by adopting routing functionality in mobile nodes. The main aim behind the developing of ad hoc networking is multi-hop relaying. Wireless Ad hoc networks or infrastructure less networks are very easy to establish by using radio waves as transmitting medium without the requirements of any other equipment or infrastructure. In such a network mobile nodes can be moved and organized freely in an arbitrary way. This dynamic connectivity of nodes allow mobile ad hoc network to be organized any where any time. Loads of research work and efforts have been done since last decade to provide support and solution to different problems and challenges related to mobile ad hoc networks. But still the fast growing technology needs attention in many areas such as routing, bandwidth, security, power consumption, collisions, simulations, and topology control due to moving nodes especially for achieving 4 generation environment and resulting best QOS's. Sprint offers a 3G/4G connection plan, currently available in select cities in the United States. It delivers rates up to $10 \mathrm{Mbit} / \mathrm{s}$. I have proposed three routing protocols (DSR, AODV, TORA) with different range, frequencies and parameters towards the contribution for best performance towards 4 generation cellular networks with an extension of ad hoc networks through a gateway i-e wireless routers or iphone etc. This has a very good contribution using OPNET simulator for best results on Real Time basis towards $4 \mathrm{G}$ cellular networks.
\end{abstract}

\section{General Terms}

Performance comparison, ad-hoc networks, Protocols, Quality of services.

\section{Keywords}

4G, DSR, AODV, TORA, OPNET simulator.

\section{INTRODUCTION}

The 4G system was originally envisioned by the Defense Advanced Research Projects Agency (DARPA). The DARPA selected the distributed architecture, end-to-end Internet protocol (IP), and believed at an early stage in peer-to-peer networking in which every mobile device would be both a transceiver and a

router for other devices in the network eliminating the spoke-andhub weakness of $2 \mathrm{G}$ and $3 \mathrm{G}$ cellular systems. Since the $2.5 \mathrm{G}$
GPRS system, cellular systems have provided dual infrastructures: packet switched nodes for data services, and circuit switched nodes for voice calls. In $3 \mathrm{G}$ and $4 \mathrm{G}$ systems, the circuit-switched infrastructure is abandoned, and only a packet-switched network is provided. This means that traditional voice calls are replaced by IP telephony. Good support for mobility, High performance mobile data is possible at speeds of up to $350 \mathrm{~km} / \mathrm{h}$, or even up to $500 \mathrm{~km} / \mathrm{h}$, depending on the frequency band used. A large amount of the work is aimed at simplifying the architecture of the system, as it transits from the existing UMTS circuit plus packet switching combined network, to an all-IP flat architecture system. Much of the standard addresses upgrading $3 \mathrm{G}$ UMTS to $4 \mathrm{G}$ mobile communications technology, which is essentially a mobile broadband system with enhanced multimedia services built on top.

For best QOS I have proposed on-demand routing protocols in hard real time basis for timely delivery of data packets in an autonomous system. As clear form the name on-demand routing also called reactive routing protocols, these protocols do not exchange routing information periodically but use flooding method to obtain information when required for a node to send data packet. The host node which needs to transmit packets to destination in network, broadcasts a route request to all nodes in network. The host node will be waiting for reply of the nodes in the network to provide a path to destination before transmitting packets. Reactive protocol performs two main functions in Ad hoc network routing, i.e. route discovery and route maintenance. The route discovery function of protocol is responsible for detecting of new route to forward packets when required by a node. Route maintenance function of reactive protocol is responsible for detecting of broken links and repairing of an existing route. The route discovered by global search process are then stored for future use until they become invalid and deleted because of link failure or topological changes. The route request and selection process leads to significant delay in initial setup of transmitting packets. Therefore real time application using on-demand routing protocols requires transmission of significant amount of control overhead due to global or flooding search method. Reactive protocols are bandwidth efficient and reduce storage space as it does not need to keep unnecessary network topology information up-to-date. The common reactive routing protocols used by MANET are DSR, AODV, TORA, ABR, SSA, LAR, FORP, LMR, ROAM and PLBR.

The maturity of wireless mobile technologies and the evolution of different applications provide a reason for the introduction of QoS in wireless ad hoc networks. The goal of QoS routing in MANET 
is to select routes with sufficient resources for data packets with QoS requirements to increase possibility that network will be capable of supporting and maintaining them. Here I proposed two reactive routing protocols along with simulation based results for efficient and accurate data delivery on timely basis using OPNET simulator V.12. In order to judge the performance of on-demand routing protocols one needs to know different metrics to deliver a greater percentage of originated data packets where there is node mobility occur. Both Dynamic source Routing (DSR), Ad-hoc ondemand Distance Vector routing protocol (AODV) and Temporally Ordered Routing Algorithm (TORA) are on-demand protocols whose basic characteristic is demonstrated in the shape of its overhead. Some simulation parameters and results are illustrated here for optimization techniques to achieved and improve performance and to reduce overhead.

\section{OVERVIEW OF ROUTING PROTOCOLS}

To discuss in details the current Mobile Ad Hoc Network (MANET) routing protocols concept as described by Internet Engineering Task Force (IETF) MANET working group as well as IEEE 802.11. In particular, Ad Hoc On-Demand Distance Victor (AODV) Routing, Dynamic Source Routing (DSR), and Temporally Ordered Routing Algorithm (TORA) are discussed along with a review of previous research implementation of these routing protocols.

\subsection{Ad-Hoc on-demand distance vector routing protocol (AODV)}

AODV routing algorithm is a routing protocol design for mobile Ad-hoc networks and is using on-demand routing approach for establishment of route between nodes. As it uses on-demand routing therefore it built route to transmit data packets when the source node desired and is trying to maintain established route as long as they are needed. AODV protocol has quality to support unicast, multicast and broadcast routing with loop free, self starting and scalable characteristics. AODV protocol routes data packets between mobile nodes of ad hoc network. This protocol allows mobile nodes to pass data packets to required destination node through neighbor's node which cannot directly communicate. Nodes of network periodically exchange information of distance table to their neighbors and ready for immediate updates. AODV protocol is responsible to select shortest and loop free route from table to transfer data packets. In case of errors or changes in selected route, AODV is able create a new route for the rest of transmission of establishment and maintenance.

\subsection{Dynamic source Routing (DSR)}

Dynamic Source Routing (DSR) Protocol is an on-demand routing protocol developed at Carnegie Mellon university Pittsburgh USA for use of multi-hop wireless mobile ad hoc networks. DSR routing protocol is designed for mobile ad hoc network to keep features of both on-demand routing protocol and source routing protocol. DSR protocol performing as on-demand routing establishes a route between source and destination node when source node wants to send data packets. In source routing, as clear from name DSR controls route through source node and data packets are sends only on those route for which source node provide information [5], like other on-demand reactive routing protocols, routing of data packets in DSR protocol between mobile nodes of ad hoc network is based on request/reply method. DSR control the wastage of bandwidth by eliminating need of periodic table updating. As discussed earlier that DSR protocol can establish a route to destination through source routing, therefore it does not require transmission of periodic hello message by a node to inform its neighbor about his presence [1]. Attractive point of DSR source routing protocol is that intermediate nodes of ad hoc network do not need to keep route information. The path is clearly defined in data packet of source node. DSR routing protocol supports uni-directional communication between mobile nodes [3]. In mobile wireless ad hoc network communication between mobile nodes through DSR routing protocol is achieved by two phases: Route establishment and maintenance.

\subsection{Temporally Ordered Routing Protocol (TORA)}

TORA is a source initiated on-demand routing protocol presented by Park and Corson in 1997 for wireless mobile ad hoc network. TORA is an efficient, highly adaptive, and scalable routing protocol based on link reversal algorithm. TORA provide multiple routes to transmit data packet between source and destination nodes of mobile ad hoc network. TORA is an adaptive routing protocol for multi-hop networks that contains different attributes such as; distributed execution, loop free and multi-path routing. This routing protocol is designed to reduce communication overhead by adapting local topological changes in ad hoc network. Another smart function of TORA routing protocol is the localization of control packets to a small region (set of nodes) near the occurrence of a topological changes due to route break. Therefore each node of network needs to contain its local routing and topology information about adjacent (one hop) nodes [9]. TORA is a highly adaptive routing protocol which provides loop free routing of data packets in ad hoc network at every instant. Each node of network has capability to contain information about its local topology changes and regular coordination with their neighbor nodes in order to detect partitions occurs in network. TORA has three basic route functions: Establishment, Maintenance, and Erasing. In TORA routing protocol, each node has capable to contain a structure of network describing node's height and status of all connected links. The importance of these heights in TORA routing protocol are that a node in network may only forward data packets downstream instead of upstream. Each node height metric is represented by quintuple $(\boldsymbol{\tau}, \mathbf{o i d}, \mathbf{r}, \boldsymbol{\delta}, \mathbf{i})$ which includes the following values.

$\begin{array}{ll}\boldsymbol{\tau} & : \text { Logical Time of a link failure } \\ \text { oid } & : \text { Unique ID of the node that defined the reference level } \\ \mathbf{r} & : \text { Reflection indicator bit } \\ \boldsymbol{\delta} & : \text { Propagation ordering parameter } \\ \mathbf{i} & \text { : Unique ID of the node }\end{array}$

\section{QUALITY OF SERVICES IN MANETS}

The widely accepted definition of QoS is defined by the consultative committee for international telephony and telegraph (CCIT) recommendation E.800 as "the collective effect of service 
performance which determines satisfaction degree of a service user". The maturity of wireless mobile technologies and the evolution of different applications provide a reason for the introduction of QoS in wireless ad hoc networks. The goal of QoS routing in MANET is to select routes with sufficient resources for data packets with QoS requirements to increase possibility that network will be capable of supporting and maintaining them. The following figure 5 shows the QoS components. Achieving QoS in mobile ad hoc network corresponds to a real need and is difficult as compared with traditional wired networks. QoS is essential element in routing which informs source node about successful availability of destination node. QoS guarantees in mobile ad hoc networks.

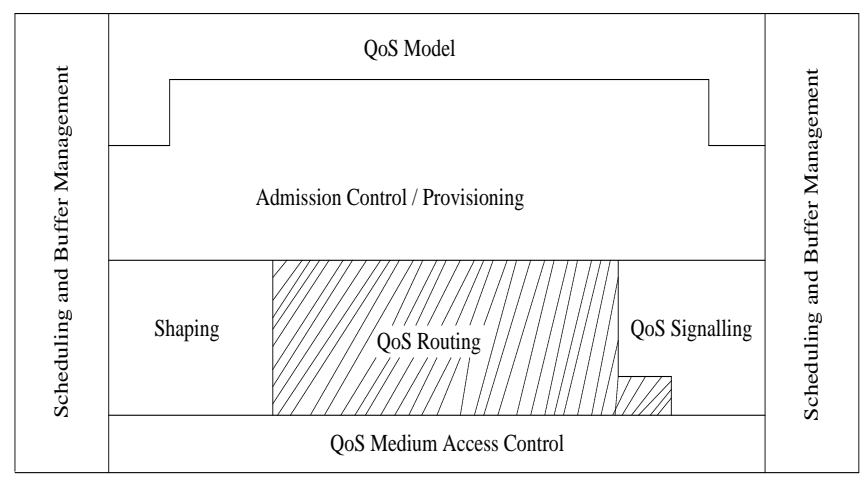

Figure 1. QOS components in routing protocol

\section{SIMULATION ENVONMENT}

Conducting real world mobile ad hoc network experiments for researchers are difficult and costly. Therefore MANET research communities commonly rely on computer simulation to evaluate and analyze their experiments on different routing protocols for dynamic ad hoc networks. The simulation results are far from perfect real environment but still provide a better understanding. Simulation is the process of designing a model of real system and conducting experiments for purpose of understanding behavior and verification of proposed system control at different stages. The selection of good simulation tool helps a lot in better testing of proposed mechanism in several different possible environments. The emerging MANET routing algorithm studies shows that some sensitive simulation parameters affect simulation results. In this paper the simulation result of two previously published MANET routing protocols AODV, DSR and TORA are presented using TCP based application. AODV, DSR and TORA are most popular MANET on-demand routing protocols. The analysis of proposed protocols is done using the MANET model in OPNET simulator.

\subsection{MANET Node Model Structure of OPNET}

Routing protocols AODV, DSR, and TORA are implemented at IP layer in MANET model structure as shown in the following figure. Models of AODV, DSR, TORA, and OLSR are available in OPNET version.12, to explains node model architecture of MANET nodes.

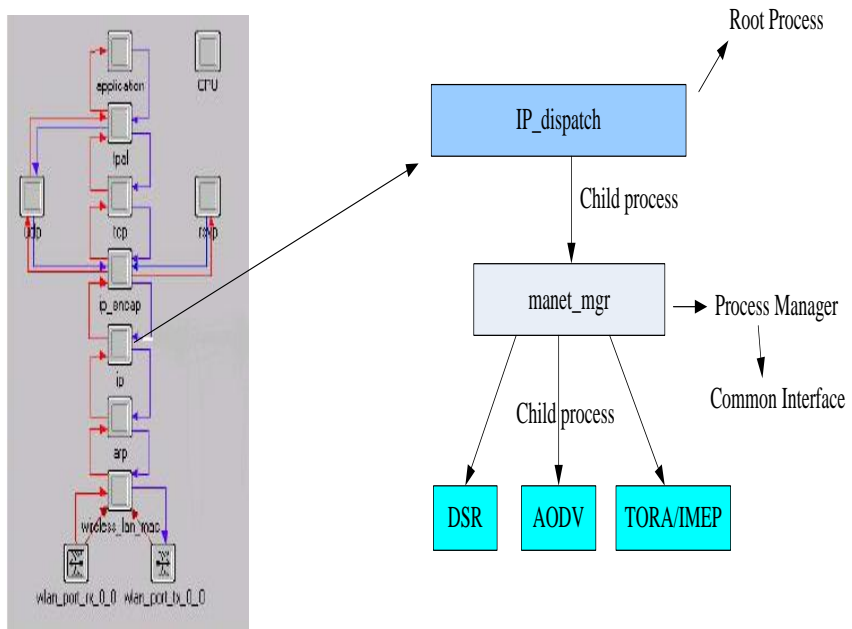

Figure 2. MANET node model structure (OPNET model)

Ip_dispatch is root process for IP in MANET node and creates a child process manet_mgr. The manet_mgr function as manager process and provides a common interface to multiple mobile ad hoc routing protocols in OPNET. The manet_mgr is further child process for required MANET protocol as configured in parameter in the proceeding table. 1 of section 4.2 .

\subsection{Analysis and Simulation Results}

Here I explain how to model and simulate three different routing protocols AOD, DSR and TORA in ad hoc network with different parameters in table1 using MANET models and The main parameters that effect mobility in Ad Hoc Network are maximum speed of mobile host nodes and pause time between each moving. In this paper I discuss different phases such as design, simulation, data collection, and analysis. In this simple scenario of ad hoc network the following parameters are used for simulation purpose.

Table 1. Simulation parameters

\begin{tabular}{|l|l|}
\hline Routing Protocols & $\begin{array}{l}\text { AODV, DSR, and } \\
\text { TORA }\end{array}$ \\
\hline Number of wireless nodes & 50 nodes \\
\hline Movement space & $\begin{array}{l}45000 \mathrm{~m} \quad \mathrm{x} \\
35000 \mathrm{~m}\end{array}$ \\
\hline Maximum speed & 2,10, and $20 \mathrm{~m} / \mathrm{s}$ \\
\hline Maximum pause time & 0 and 200s \\
\hline Transmission rates & $\begin{array}{l}2,5, \quad \text { and } 10 \\
\text { packets } / \mathrm{s}\end{array}$ \\
\hline Packet size & 512 bytes \\
\hline Simulation time & $300 \mathrm{~s}$ \\
\hline
\end{tabular}

The simulated scenario evaluates the performance of important TCP parameters for AODV based 50 nodes network. All nodes in the network are configured to run AODV protocol and FTP sessions. In simulation process different AODV parameters are used as suggested by RFC and WLAN data rate. A single TCP connection is established between wireless nodes of scenario. Two different self mobility trajectories are defined for mobile. The results shows amount of routing traffic generated, route 
discovery time and the number of hops per route, TCP/IP traffic, no; of RREQ and RREP packets and FTP download and upload time. Simulation of mobile ad hoc network consist of thirty nodes was run for 300 seconds and the following results were generated.

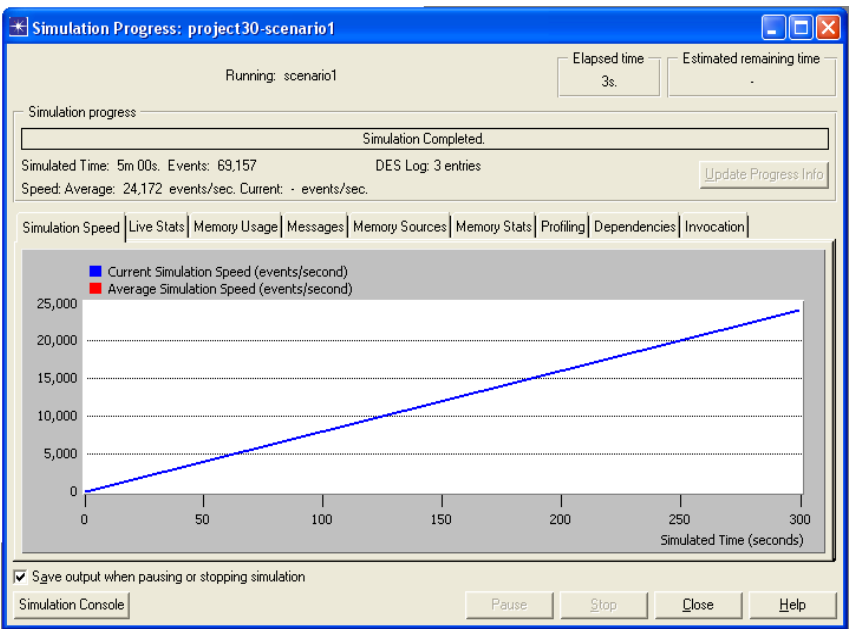

Figure 3. Simulation progress report for MANET routing protocols in collaboration with cellular network (PDA).

\section{Proposed performance based Routing Model}

From For best routing between wireless nodes of ad hoc network a number of qualitative properties is desirable. For best and effective routing loop freedom is very necessary for data packets to avoid collision and waste of time. MANET uses time to live (TTL) to avoid such loops, but more structured approach is required to get best results. The MANET delay effects on routing very badly, as the mobile nodes are battery powered and scarce memory. The routing protocol needs to be routed intelligently and utilize network bandwidth and energy resources in a better way in case of network route delay. One of the important characteristics of MANET is dynamic topology based on node mobility. Mobile nodes in ad hoc network are free to change position frequently, therefore routing protocol needs to quickly adopt topology changes. Due to dynamic mobility and limited energy resources a unique wireless node in MANET cannot be trusted for auto configuration in case of break down. Therefore support of distributed operation is required by routing protocols to solve such type of problems. Due to lack of physical security, MANET protocols are highly exposed to different types of attacks. In MANET it is very easy for attacker to disturb network traffic, corrupt packet header, change addresses of routing messages, and increase traffic to waste bandwidth. To avoid all these threats a sufficient security protection is highly desirable in all MANET routing protocols. In the $3 \mathrm{D}$ environment of performance comparison of MANETS Routing Protocols traffic sent and received is shown in figure 4 . Thus the combined performance of AODV, DSR and TORA routing protocol could be best solution for routing in MANET instead of separate performance.

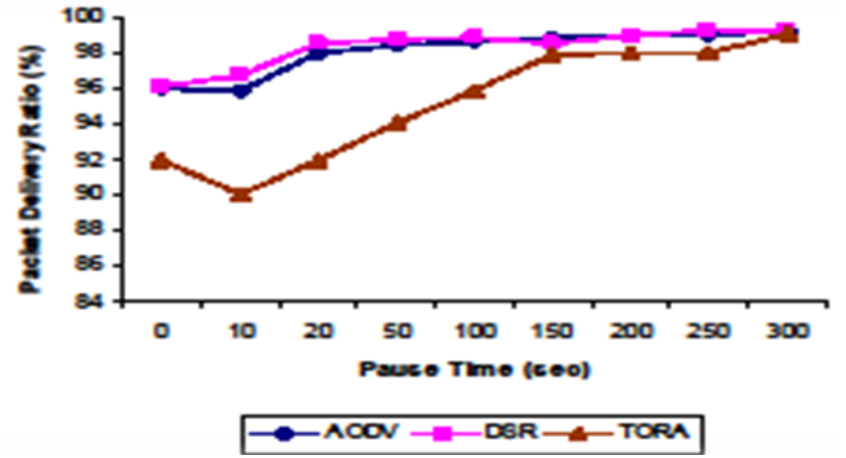

Figure 4. Performance comparison and delivery ratio in MANETS

\section{Target Audience of MANET}

The routing protocol needs to be routed intelligently and utilize network bandwidth and energy resources in a better way in case of network route delay. The simulated scenario shows that routing overhead for the routing protocols heavily depends on node mobility. AODV sends many small routing control packets while DSR sends less but bigger control packets during transmission of data packets. DSR is more useful in smaller networks with less mobility and usage of AODV and TORA are more appropriate in ad hoc networks with a higher mobility and data transfer rate. The simulations results and analysis have shown that mobile ad hoc network definitely needs more precise routing protocols to support increase mobility. I came to the conclusion that for small network DSR is suitable and for medium network AODV is appreciate able and also for large network TORA is very good for data delivery ratio, in order to extend autonomous system to hybrid networks either for best QOS or VOIP i-e enhancing 3G towards $4 \mathrm{G}$, these on demand protocols may contribute for higher data delivery ratio to obey the $4 \mathrm{G}$ data throughput and provide a QOS in ad hoc mode. A vision of $3 \mathrm{G} / 4 \mathrm{G}$ is shown in figure 4 [9].

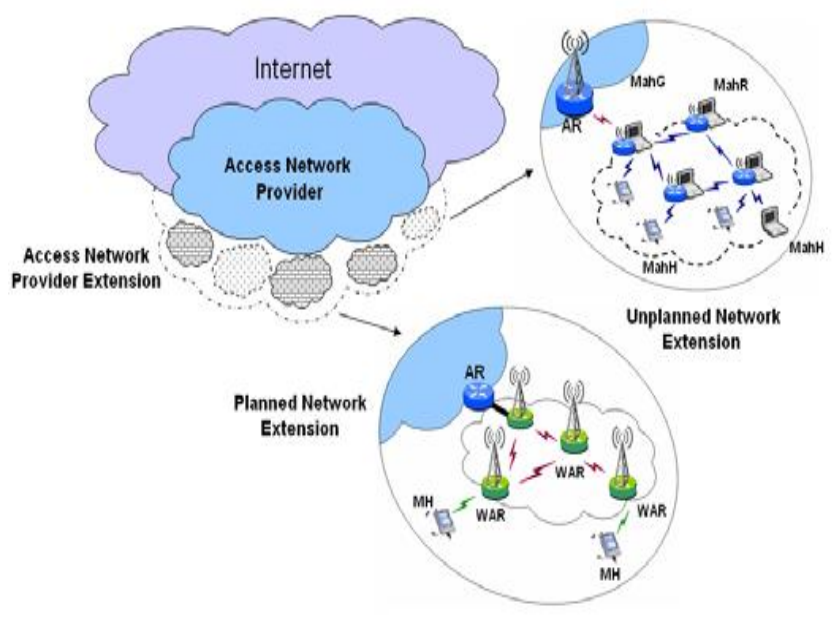

Figure 4. Planned and Unplanned extension of access network providers with ad hoc networks. 


\section{Content}

This paper will cover the following topics:

- Comprehensive differences in mobile ad-hoc networks topology for different data frequencies in each source, intermediate and destination nodes respectively.

- Overview of MANET and Reactive Routing protocols.

- Methodologies for providing best QOS using ondemand routing protocols.

- Important open research issues in mobile ad-hoc networks for efficient and best performance.

- Comparison of DSR,AODV and TORA Routing protocols for small and large networks in an autonomous system.

- A step towards $4 \mathrm{G} /$ enhancing in $3 \mathrm{G}$ for best QOS.

\section{CONCLUSION}

In this paper we present the simulation results of three wireless routing protocols under scalable mobile condition for the correctness and efficiency in fifty nodes scenario, mobile nodes which effect greatly in routing and size of the network, traffic load, and delay. To present the simulation results of three wireless routing protocols under scalable mobile condition for the correctness and efficiency in $3 \mathrm{G} / 4 \mathrm{G}$. In this scenario, mobile nodes which effect greatly in routing and size of the network, traffic load, and delay affects both AODV, DSR and TORA routing protocols and also study cases of TORA for better QOS support. DSR routing protocol is best for small network but not efficient for large networks with many mobile nodes and high load in terms of traffic and delay which will increase overhead. In such situation AODV routing protocol is ideal because of its hopby-hop routing and also for large network TORA routing protocol is good for large network. Here we explain how to model and simulate three different routing protocols AODV, DSR and TORA. Thus the combined performance of these routing protocols could be best solution for routing in MANET instead of separate performance of AODV, DSR and TORA. Enhancing of MANET for $4 \mathrm{G}$ is future work to work in distributed environment through mobile gateways (i-e PDA's, iphone, Harris wireless Technology) for best results.

\section{ACKNOLEDGMENTS}

We are very pleased of professor Dr. Salim ur Rehman for their moral support, we also grateful to IJCA for exploring our ideas and research in the human planet, we are also want to thank our family for their pray.

\section{REFERENCES}

[1] Lee, S. (2000) Routing and Multicasting Strategies in Wireless Mobile Ad Hoc Networks, [Internet].University of California Los Angeles. Available from: 〈http://www.sigmobile.org/phd/2000/theses/sjlee.pdf>

[2] Nicolau, A. \& Grigoras, D. (2005) Concurrent Information Processing and Computing. $2^{\text {nd }}$ edition. Netherlands, IOS Press

[3] Tavel p.2007.modeling and simulation design.A.K.Peter Ltd Natick MA

[4] OPNET. (2007) Modeler Wireless Suite for Defence. [Internet].Availablefrom: <http://www.opnet.com/products/modeler/home.html>

[5] Lee, S. (2000) Routing and Multicasting Strategies in Wireless Mobile Ad Hoc Networks, [Internet].University of California Los Angeles. Available from:

<http://www.sigmobile.org/phd/2000/theses/sjlee.pdf>

[6] Jahangir khan, "Base on MANET the correctness and performance evaluation of DSR,AODV and TORA Routing protocols for best QOS 'International Journal of Computer Applications, Foundation of Computer Science, New York, USA. http://www.ijcaonline.org/specialissues/manets/numbe r3/1029-74.

[7] Jahangir khan, "Handover management in GSM cellular system". International Journal of Computer Applications, Foundation of Computer Science, New York, USA http://www.ijcaonline.org/archives/volume8/number12/1257 $-1763$.

[8] Jahangir khan, Dr. syed Irfan hyder. "A Comprehensive Performance Comparison of On-demand Routing Protocols in Mobile Ad-hoc Networks" . By FGCN2010 Jeju island South Korea: FGCN 2010, PartII, CCIS120, pp.354369.Springer, Heidelberg (2010) https://www.eproof.sps.co.in/lncs/ja.asp?rfp=eij hjjjhgdfe

[9] Govind Singh Tanwar , Ganesh Singh , Vishal Gour "Multimedia Streaming Technology n 4G Mobile Communication Systems" Govind Singh Tanwar et. al. / (IJCSE) International Journal on Computer Science and Engineering. Vol. 02, No. 03, 2010, 695-699

[10] Yih-Chun Hu, David B. Johnson ."Ensuring Cache Freshness in On-Demand Ad Hoc Network Routing Protocols" POMC'02, October 30-31, 2002, Toulouse, France. ACM.

[11] Marcin Szczodrak, Jinwoo Kim, and Yuncheol Baek, "4GM@4GW: Implementing 4G in the Military Mobile AdHoc Network Environment" IJCSNS International Journal of Computer Science and Network Security, VOL.7 No.4, April 2007

[12] M. Bani Yassein, M. Ould Khaoua, L. M. Mackenzie, S. Papanastasiou, A. Jamal "Improving Route Discovery in OnDemand Routing Protocols Using Local Topology Information in MANETs" PM2HW2N'06, October 2, 2006, Torremolinos, Malaga, Spain. 